\title{
Actual duration of patient-reported mucositis: Far longer than 2 to 4 weeks and may be avoidable altogether
}

\author{
Ricky Wayne McCullough ${ }^{1,2}$ \\ ${ }^{1}$ Translational Medicine Research Center of Mueller Medical International, Storrs-Mansfield, CT; \\ ${ }^{2}$ Department of Medicine \& Emergency Medicine, Veterans Administration Medical Center Teaching Hospital, Warren Alpert Medical School of Brown \\ University, Providence, RI, USA
}

The impression that oral mucositis is a brief 14-28 day-consequence of chemoradiation is misguided. Clinically significant patient-reported oral mucositis may last 46 to 102 days depending on the treatment schedule and the modality used. The process of mucositis can occur in the epithelium throughout the entire Gl tract and may possibly be avoided with the prescribed use of high potency polymerized crosslinked sucralfate (HPPCLS). Literature review of patient-reported mucositis occuring in the three common schedules of cancer treatment administration: daily radiation therapy, induction-based hematopoietic stem cell transplantation (HSCT), and multi-cycle chemo/immunotherapy (CT-IT). Review articles published in last 15 years focused on treatment-induced oral mucositis. The author reviewed 56 articles published in 15 years from 1999-2014 that focused primarily on treatment-induced oral mucositis. Only 6 were found to meet the criteria of providing patient-reported data from the beginning, throughout and following cancer treatment. For HSCT, radiation therapy, and CT-IT, despite active anti-mucositis treatment, patient-reported oral mucositis lasted 46-60 days, 70-84 days, and 68-102 days, respectively. Mucositis caused by cancer treatment, regardless of modality, lasts far longer than the oft quoted 2-4 weeks (range, 14-28 days). Patient reported mucositis persists from 46 to 102 days. This patient-based experience is the primary cause of treatment interruptions, delays or cancellations. It may be avoidable with HPPCLS that both prevents and rapidly reverses mucositis anywhere it occurs within the gastrointestinal tract.

Keywords: Mucositis, Chemotherapy, Radiation therapy, Hematopoietic stem cell transplantation, Sucralfate, Polymerization

\section{INTRODUCTION}

There are three major treatment modalities wherein mucositis arises and can affect patient's willingness to continue cancer treatment

Received: Apr 18, 2016 Accepted: May 20, 2016

Correspondence to: Ricky Wayne McCullough

Translational Medicine Research Center of Mueller Medical International, 1768 Storrs Road, Storrs-Mansfield, CT, 06268, USA

Tel: +1-860-477-0961, Fax: +1-860-477-0962

E-mail: glencopelph@aol.com

*By written permission from both author and editors, it is republished here in Clinical Oncology Journal to benefit the Korean academic oncology community. This article is based on a commentary first published by the author in International Journal of BioMedicine 2014, 4(4):194-197, entitled: Commentary Regarding Mucositis During Cancer Therapy-Duration of Mucositis.

Copyright (C) Korean Society of Surgical Oncology

This is an Open Access article distributed under the terms of the Creative Commons Attribution Non-Commercial License (http://creativecommons.org/licenses/by-nc/4.0) which permits unrestricted non-commercial use, distribution, and reproduction in any medium, provided the original work is properly cited. without interruptions, delays or cancellations. These include daily radiation therapy (RT), induction-based hematopoietic stem cell transplantation (HSCT) and multi-cycle chemo/immunotherapy (CTIT). Yet with each of this modalities, mucositis is regarded as a tolerable consequence of treatment due to its brief occurrence.

In fact the oft-repeated refrain that "mucositis is self-limited when uncomplicated by infection and typically heals within 2 to 4 weeks after cessation of cytotoxic chemotherapy" or radiation [1], is an inexact statement that can be unintentionally disarming. It gives the impression that the duration of mucositis is short therefore tolerable particularly if there is no infection. Again, when referenced in material intended for patients [2], this statement is repackaged as follows: "In patients getting chemotherapy, mucositis will heal by itself when there is no infection. Healing usually takes 2-4 weeks." To be fair, the reference continues: "Mucositis caused by radiation therapy usually lasts $6-8$ weeks", which is closer to the reality, but is still far short of the 10-14 weeks required for the mucosa and mouth throat soreness to return to baseline $[3,4]$. The impression that mucositis is a short-lived event is inaccurate, can be 
misleading and therefore detracts from appropriate clinical vigilance necessary to manage it.

Another impression given by these statements is that mucositis persisting beyond " 2 to 4 weeks" is likely due to local infection which in turn should be suspected as the main driver of the mucositis process and not the cancer therapy itself. This impression likely underlies the observation of many patients with persisting mucositis are prescribed an anti-fungal, anti-viral or antibiotic agent. However, because there is no clear evidence that antimicrobials shorten the course or lessen the severity of oral mucositis, their use is not recommended in mucositis guidelines [5]. Clearly, cancer therapy itself is the main driver of clinical mucositis.

The concept that mucositis is likely an ephemeral, self-limited process that is over in "2 to 4 weeks" serves to 'disconnect' the clinician prescribing chemo-radiation from the patient experiencing mucositis. Indeed, Fromme et al. [6] disclosed that physician reporting on adverse reactions to chemotherapy lacked both sensitivity and specificity being incongruent with patient-reported experiences. Trotti et al. [7] reviewed 33 studies examining 6,181 patients with mucositis. Only 13 reported all grades of mucositis, 9 reported grades 3-4 only and only 1 reported on the quality of life. Though the most severe forms of mucositis-Grades 3, 4-are likely to be reported, dose reductions and unplanned treatment interruptions occur more frequently with less severe mucositis (23\% with Grades $1,2)$ than with severe mucositis $(21 \%$ with Grades 3,4$)$ [8]. These disconnects remain as hidden biases that can compromise the appropriate management of mucositis. Unfortunately, the lack of clinically effective mucositis interventions bury these disconnects from view and rendering them irrelevant. Yet an unforeseen effect of these "buried" disconnects is that the advent of effective mucositis interventions will be challenged, as some clinicians may possibly be lulled into a position of indifference.

Ulcerations, erythema and the pain of mucositis patients receiving daily radiation are more likely to be noticed by the clinical staff as compared to patients receiving chemotherapy who can escape notice of the clinical staff, as they often would have navigated through the worst of their mucositis experience by the beginning of their next cycle of chemotherapy. The mouth and throat soreness of chemotherapy-induced mucositis intensifies with successive cycles, with amplification by one report, as much as 44\% [9]. Recurrent bouts of mucositis double the likelihood of dose reduction and unplanned treatment interruptions [8] which directly and negatively impacts tumor kill and thereby survival. Therefore, recognition of patient-reported duration of clinical mucositis is an essential element for clinicians to sense of urgency in addressing mucositis when it occurs.

\section{METHODS}

Review of published articles

It is difficult to find a single reference detailing patient-reported duration of clinical mucositis in context of all three major treatment scenarios. Published papers were identified under the following criteria, during the cited search period using the following search tool.

\section{Criterion for review}

Patient-reported vs. investigator-reported assessments Patient-reported assessment of mucositis was the focus of this inquiry. While investigator-reported mucositis assessments are useful, patient-reported experience of mucositis is the basis of patient-initiated treatment interruptions, delays or cancellations. This is particularly true with Grade 1 oral mucositis wherein there are no ulcerations visible to an investigator, but patient may have burning mouth syndrome that makes eating, swallowing and drinking difficult, with normal-appearing mucosa. This phenomenon of burning mouth syndrome of Grade I oral mucositis together with Grade 2 muositis is twice as likely to cause dose reductions in chemotherapy patients with mucositis compared to those without mucositis [8].

\section{Search period}

Search period of 1999 to 2014 was selected because during this period of time five anti-mucositis agents had been approved by US FDA (Gelclair, MuGard, Episil, Caphosol and Kepivance/palifermin).

\section{Search tool}

The medline/pubmed search tool was used to identify 56 articles pertaining to cancer treatment-induced mucositis.

\section{RESULTS}

Articles used to describe the duration of patient-reported mucositis

Of the 56 articles on the subject of oral mucositis, five [3,4,9-11] provided or alluded to validated methods of patient-reported duration of mucositis.

Tabulation of patient-reported duration of mucositis In practice, there are three clinical treatment scenarios of significance that are associated with the development of mucositis: (a) myeloablative conditioning in HSCT, (b) multicycle chemotherapy, and (c) radiation therapy with or without chemotherapy. Treatment-specific illustrations of the duration of patient-reported oral mucositis (PROM) are shown in Fig. 1. 

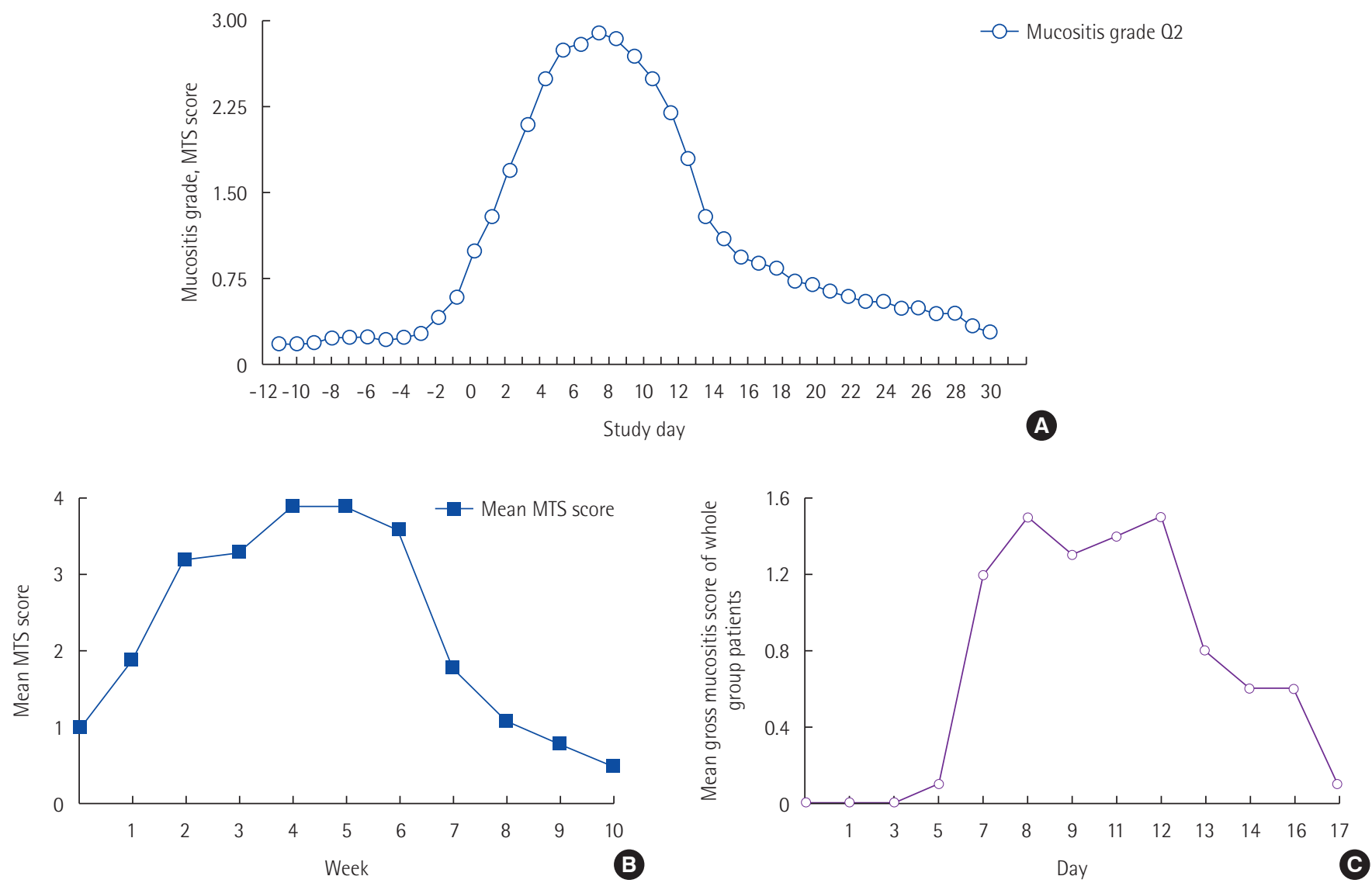

Fig. 1. Duration of patient reported oral mucositis (PROM). (A) PROM in patients undergoing hematopeietic stem cell transplantation (HSCT) (adopted from Stiff et al. [10]). (B) PROM in patients undergoing radiation for squamous cell carcinoma of head and neck (adopted from Elting et al. [4]). (C) PROM in patients undergoing chemotherapy (adopted from Chi et al [9]). MTS, mouth throat soreness.

\section{Patient-reported mucositis in HSCT}

First, for HSCT patients (Fig. 1A) typically receiving single myeloablative doses of chemotherapy and irradiation, the painful mouth throat soreness affecting eating and drinking begins on day 2, with erosions and ulcerations appearing day 5-7 of oral mucositis. The peak mouth-throat soreness spans an additional 2-3 days before slowly declining over the following 18 days [10], with an extrapolated return to baseline by day 46 . Kushner et al. [11] reported that patient-reported return to baseline for this cohort may take up to 60 days following myeloablative therapy. Nearly 100\% of these patients experience grade 3 to 4 oral mucositis.

\section{Patient-reported mucositis in radiation with or without chemotherapy}

Secondly, patients receiving the standard 6 to 7 week (range, 42-49 days) radiation treatment for head and neck cancer can experience multiple grades mucositis over 70 day period (Fig. 1B) [3,4]. With combined chemo-radiation, clinical mucositis will persist up to 84 days, and on occasion beyond [4]. Two years following chemo-radi- ation, a prior episode of mucositis-related inflammation contributes to chronically persistent difficulty in swallowing (confirmed by video fluoroscopy) that negatively impacts the patient-reported quality of life [12].

Patient-reported mucositis in multi-cycle chemotherapy Lastly, and perhaps the most complicated patient group, is that of patients receiving single doses of multiple chemotherapeutic agents in 4 to 6 cycles. In this patient group mucositis experience varies based on the time of exposure to, and the type of agent used. In a triple regimen of cisplatin, fluorouracil and leucovorin administered over a four-day continuous infusion, the resultant episode of mucositis persisted over a period of 17 to 21 days per cycle (Fig. 1C) [9]. This is likely the minimal time duration of mucositis. Of course, when cycles are given weekly (as with certain regimens), the period of mucositis can be extended. Generally, chemotherapy is administered in 4 to 6 cycles, with the majority of cycles repeating every 14 to 21 days, and a minority of cycles repeated every 7 or 28 days [13]. Regardless, as mentioned earlier, the intensity of mucositis ulcer- 

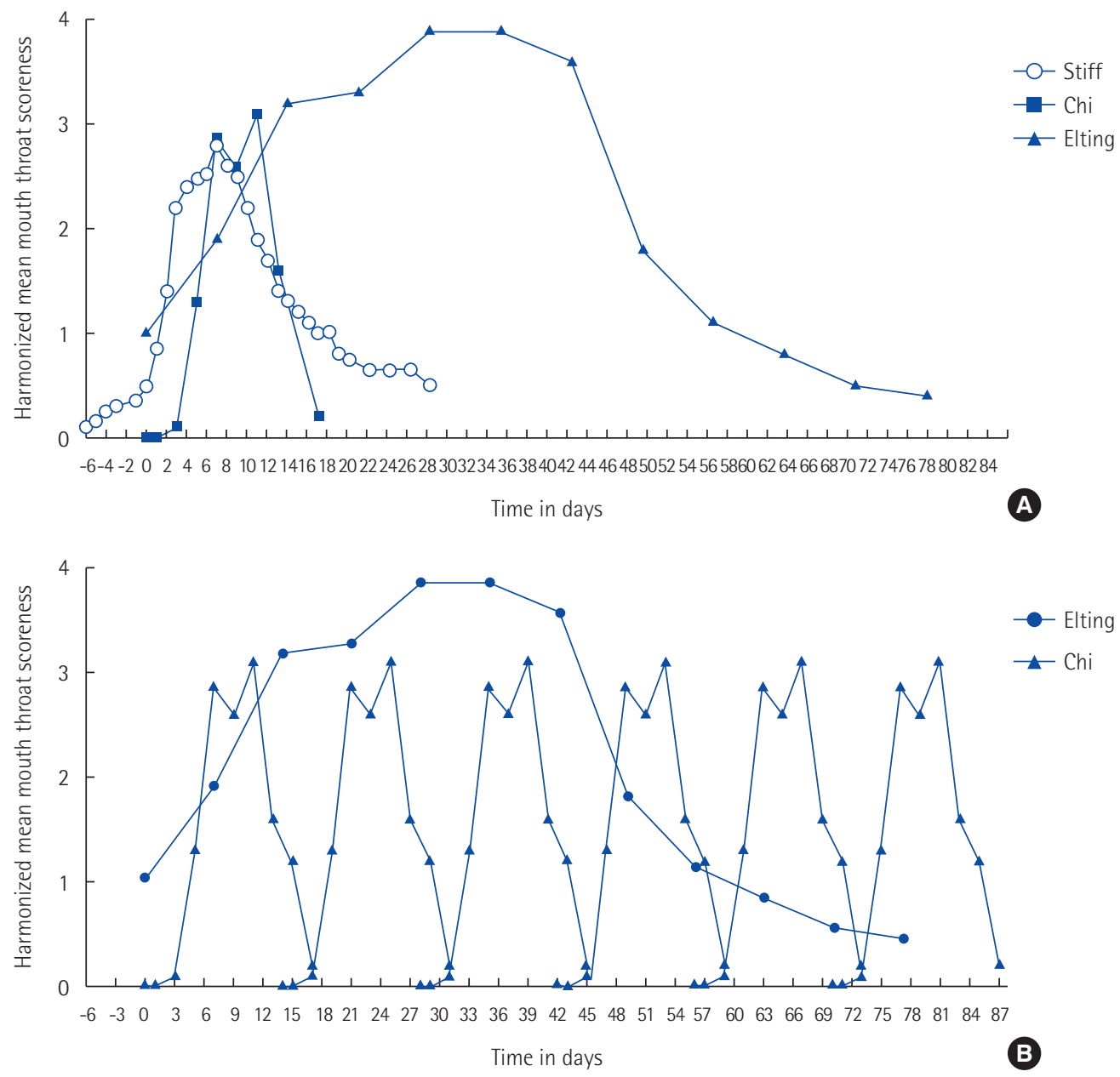

Fig. 2. Composite of patient reported oral mucositis (PROM). (A) Composite graph of PROM with harmonized mean mucositis score (adopted from Stiff et al. [10], Elting et al. [4], and Chi et al. [9]). (B) Composite graph of PROM with harmonized mean mucositis score following 42 days radiation and 6 cycles of chemotherapy repeated every 14 days (adopted from Elting et al. [4] and Chi et al. [9]).

Table 1. Duration in days of clinically significant oral mucositis

\begin{tabular}{lccc}
\hline Therapy & Days of mucositis & Percent patients affected & Reference \\
\hline Myeloablative therapy HSCT & $46-60$ & 1.6 & Stiff et al. [10], Kushner et al. [11] \\
Radiation \pm chemotherapy & $>70-84$ & 13.8 & Elting et al. [3,4,8] \\
Multiple cycle chemotherapy & $68-102$ & 84.6 & Chi et al. [9] \\
\hline
\end{tabular}

HSCT, hematopoietic stem cell transplantation.

ation and patient-reported pain amplifies with successive cycles [9].

\section{Harmonization of data points}

Harmonizing data points in terms of a mean mucositis score (for the $y$-axis) over a period of 84 days (as the $x$-axis) provides a perspective on the duration (Fig. 2) of clinical (symptomatic) mucositis. Fig. 2A compiles all three scenarios offering a contrast of the experience of mucositis in terms of time. For patients receiving multiple cycles of chemotherapy (Fig. 2B), recurrent episodes of ever-worsening mucositis can be daunting.
By far the longest periods of mucositis experienced are in patients receiving combined chemo-radiation where symptoms can persist beyond 80 days, while the shortest period is among patients receiving myeloablative therapy for HSCT with symptoms that extrapolate out to 46 days. Of the 400,000 patients [14] developing oral mucositis annually, approximately 55,000, or 14\%, are patients with head and neck cancer many of them requiring combined chemo-radiation [15]. An additional 6,300 [16], or 1.6\% of all oral mucositis patients underwent HCST. The remaining 84.4\% largely consists of patients receiving multiple cycles of chemotherapy who de- 
velop mucositis on successive cycles [9].

Actual duration of patient-reported mucositis

Thus, the actual duration of mucositis (Table 1) is as long as 46 to 60 days in HSCT patients, more than 84 days in patients receiving combined chemo-radiation, and, in the vast majority of patients receiving four to six cycles of chemotherapy, there can be 68 to 102 days of clinical mucositis to endure.

\section{DISCUSSION}

It is the subjective experience of mucositis that drives patient-initiated treatment interruptions, delays and cancellation. Unplanned treatment delays lead to early recurrence of loco-regional disease and lower 5 year survival [17-20]. Considered for this perspective, mucositis is an excessive burden borne throughout treatment for those who develop it. It severely impacts their ability to swallow, eat and drink. This limitation directly causes weight loss, dehydration, ER visits, hospitalizations and increased cost of care $[3,4]$.

Based on the clinical outcomes of a post-market mucositis registry involving a recently FDA cleared therapeutic intervention, mucositis may possibly be avoidable altogether [21]. A sucralfate-based medical device, described as polymerized cross-linked sucralfate, differentiates itself from other interventions by an association with mucositis prevention and rapid reversal in patients undergoing chemo-radiation $[22,23]$. In a post-market mucositis registry, 27 patients were successfully treated, experiencing rapid reversal of oral mucositis in 2-3 days. Six patients were successfully prevented from developing mucositis averting surgical insertions of prophylactic gastrostomy feeding tubes [24]. The observational data from the medical device registry demonstrated a positive Glasziou treatment effect [25], an effect wherein efficacy is secured by the sheer magnitude of the intervention's treatment response. In chemo-radiation mucositis, a time of 2-3 days to achieve a mucositis-free state, compared to a customary time of 70-84 days for placebo or the natural course of the disease, establishes a positive Glasziou treatment effect. The rate of symptom/sign improvement associated with an intervention exceeds 1,000 times that expected from placebo or any other intervention. The confounding biases generally controlled in randomized (non-observational) trial, are not of sufficient strength to alter the treatment effect that is 1,000 times greater than placebo [25].

\section{CONCLUSION}

In the supportive care of cancer treatment patients, the duration of mucositis is not a trivial point. Considering the real-world timing of common cancer treatment scenarios, optimal treatment outcomes heavily depends on the maintenance of required dose-intensity, that is, the set number of treatment doses per period of time. The duration of mucositis experienced by patients can impact oncologists' ability to maintain the dose-intensity required for cure. Oncologists should be aware that 14-28 days is not the average duration of patient-reported mucositis. Regardless of the use of agents such as Gelclair, Episil, MuGard, Caphosol and even palifermin/Kepivance, patients undergoing HSCT will experience mucositis for 46 to 60 days, patients receiving-radiation for head and neck cancer (with or without chemotherapy) will likely suffer mucositis for more than 80 days, and those receiving four to six cycles of chemotherapy or immunotherapeutics will endure 68 to 102 days or 17 days per cycle of mucositis cumulatively throughout their treatment. Notwithstanding other sequelae of mucositis (e.g., systemic infections, dose reductions, increased costs, altered survival), this fact alone-the actual duration of patient-reported mucositis-begs the use of interventions that could possibly reverse this added burden on a disease process (cancer) largely viewed as life-threatening in most patients.

\section{CONFLICT OF INTEREST}

Author is an employee and own stock in Mueller Medical International LLC, owner of ProThelial ${ }^{\mathrm{TM}}$.

\section{ACKNOWLEDGMENTS}

This study was supported by MMI Academic Grant 5-2014-08-15.

\section{REFERENCES}

1. National Cancer Institute. Oral complications of chemotherapy and head and neck radiation (PDQ): Health Professional Version [Internet]. Bethesda (MD): National Cancer Institute at the National Institutes of Health; 2016 [cited 2016 Jun 22]. Available from: http://www.cancer.gov/cancertopics/pdq/supportivecare/ oralcomplications/HealthProfessional.

2. Medline Plus. Oral mucositis [Internet]. Bethesda (MD): U.S. National Library of Medicine; 2014 [cited 2016 Jun 22]. Available from: http://www.nlm.nih.gov/medlineplus/ency/patientinstructions/000047.htm.

3. Elting LS, Keefe DM, Sonis ST, Garden AS, Spijkervet FK, Barasch A, et al. Patient-reported measurements of oral mucositis in head and neck cancer patients treated with radiotherapy with or without chemotherapy: demonstration of increased frequency, severity, resistance to palliation, and impact on quality of life. Cancer 
2008;113:2704-13.

4. Elting LS, Cooksley CD, Chambers MS, Garden AS. Risk, outcomes, and costs of radiation-induced oral mucositis among patients with head-and-neck malignancies. Int J Radiat Oncol Biol Phys 2007;68:1110-20.

5. Saunders DP, Epstein JB, Elad S, Allemano J, Bossi P, van de Wetering MD, et al. Systematic review of antimicrobials, mucosal coating agents, anesthetics, and analgesics for the management of oral mucositis in cancer patients. Support Care Cancer 2013;21: 3191-207.

6. Fromme EK, Eilers KM, Mori M, Hsieh YC, Beer TM. How accurate is clinician reporting of chemotherapy adverse effects? A comparison with patient-reported symptoms from the Quality-of-Life Questionnaire C30. J Clin Oncol 2004;22:3485-90.

7. Trotti A, Bellm LA, Epstein JB, Frame D, Fuchs HJ, Gwede CK, et al. Mucositis incidence, severity and associated outcomes in patients with head and neck cancer receiving radiotherapy with or without chemotherapy: a systematic literature review. Radiother Oncol 2003;66:253-62.

8. Elting LS, Cooksley C, Chambers M, Cantor SB, Manzullo E, Rubenstein EB. The burdens of cancer therapy: clinical and economic outcomes of chemotherapy-induced mucositis. Cancer 2003;98:1531-9.

9. Chi $\mathrm{KH}$, Chen $\mathrm{CH}$, Chan WK, Chow KC, Chen SY, Yen SH, et al. Effect of granulocyte-macrophage colony-stimulating factor on oral mucositis in head and neck cancer patients after cisplatin, fluorouracil, and leucovorin chemotherapy. J Clin Oncol 1995;13: 2620-8.

10. Stiff PJ, Emmanouilides C, Bensinger WI, Gentile T, Blazar B, Shea $\mathrm{TC}$, et al. Palifermin reduces patient-reported mouth and throat soreness and improves patient functioning in the hematopoietic stem-cell transplantation setting. J Clin Oncol 2006;24:5186-93.

11. Kushner JA, Lawrence HP, Shoval I, Kiss TL, Devins GM, Lee L, et al. Development and validation of a Patient-Reported Oral Mucositis Symptom (PROMS) scale. J Can Dent Assoc 2008;74:59.

12. Hunter KU, Schipper M, Feng FY, Lyden T, Haxer M, Murdoch-Kinch

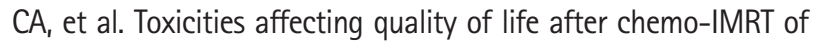
oropharyngeal cancer: prospective study of patient-reported, observer-rated, and objective outcomes. Int J Radiat Oncol Biol Phys 2013;85:935-40.

13. D'Olimpio J, Adams VR. Oral mucositis: causative regiments and pathways for treatment [Internet]. New York: Published for Clinical Oncology by McMahan Publishing; 2008 [cited Jun 22]. Availavle from: www.clinicaloncology.com/download/pg08114_ mucositis_WM.pdf.
14. Brown CG, Wingard J. Clinical consequences of oral mucositis. Semin Oncol Nurs 2004;20:16-21.

15. American Cancer Society Inc. Cancer facts \& figures 2014 [Internet]. Atlanta (GA): American Cancer Society; 2016 [cited 2016 Jun 22]. Available from: http://www.cancer.org/research/cancerfactsstatistics/cancerfactsfigures2014/.

16. US Dept. of Health and Human Services. Blood cell transplant: donor registry transplant data [Internet]. Rockville (MD): Health Resources and Services Administration; [cited 2016 Jun 22]. Available from: http://bloodcell.transplant.hrsa.gov/research/ transplant_data/registry_tx_data/index.html.

17. Foote M. The importance of planned dose of chemotherapy on time: do we need to change our clinical practice? Oncologist 1998; 3:365-8.

18. Russo G, Haddad R, Posner M, Machtay M. Radiation treatment breaks and ulcerative mucositis in head and neck cancer. Oncologist 2008;13:886-98.

19. Suwinski $R$, Sowa $A$, Rutkowski T, Wydmanski J, Tarnawski R, Maciejewski $\mathrm{B}$. Time factor in postoperative radiotherapy: a multivariate locoregional control analysis in 868 patients. Int J Radiat Oncol Biol Phys 2003;56:399-412.

20. Groome PA, O'Sullivan B, Mackillop WJ, Jackson LD, Schulze K, Irish JC, et al. Compromised local control due to treatment interruptions and late treatment breaks in early glottic cancer: population-based outcomes study supporting need for intensified treatment schedules. Int J Radiat Oncol Biol Phys 2006;64:1002-12.

21. McCullough RW. High potency polymerized cross-linked sucralfate manages both oral and alimentary mucositis simultaneously. Proceedings of the June 2014 MASCC International Symposium: 2014 Jun 26-28; Miami, USA.

22. McCullough RW. New category of evidence should permit the multinational association of support in cancer care (MASCC) to review polymerized cross-linked sucralfate paste (ProThelial ${ }^{\mathrm{TM}}$ ) for mucositis guidelines. Oncol Discov 2014;2. http://dx.doi.org/10.7243/20526199-2-1.

23. McCullough RW. High-potency sucralfate prevents and rapidly reverses chemo-radiation mucositis in a patient with stage $4 \mathrm{~b}$ head and neck cancer. World J Transl Med 2013;2:13-21.

24. McCullough RW. Prothelial ${ }^{\mathrm{TM}}$ (polymerized cross-linked high potency sucralfate): medical device therapy for treatment and prevention of mucositis. Eur J Res Med Sci 2014;2:30-58.

25. Glasziou P, Chalmers I, Rawlins M, McCulloch P. When are randomised trials unnecessary? Picking signal from noise. BMJ 2007; 334:349-51. 